\title{
Time-gated topographic LIDAR scene simulation
}

\author{
Scott D. Brown ${ }^{a \dagger}$, Daniel D. Blevins ${ }^{b \neq}$ and John R. Schott ${ }^{a}$ \\ ${ }^{a}$ Digital Imaging and Remote Sensing Laboratory \\ Chester F. Carlson Center for Imaging Science, Rochester Institute of Technology \\ ${ }^{b}$ U.S. Air Force \& Rochester Institute of Technology \\ 54 Lomb Memorial Drive, Rochester, NY 14623
}

\begin{abstract}
The Digital Imaging and Remote Sensing Image Generation (DIRSIG) model has been developed at the Rochester Institute of Technology (RIT) for over a decade. The model is an established, first-principles based scene simulation tool that has been focused on passive multi- and hyper-spectral sensing from the visible to long wave infrared (0.4 to 14 $\mu \mathrm{m})$. Leveraging photon mapping techniques utilized by the computer graphics community, a first-principles based elastic Light Detection and Ranging (LIDAR) model was incorporated into the passive radiometry framework so that the model calculates arbitrary, time-gated radiances reaching the sensor for both the atmospheric and topographic returns. The active LIDAR module handles a wide variety of complicated scene geometries, a diverse set of surface and participating media optical characteristics, multiple bounce and multiple scattering effects, and a flexible suite of sensor models. This paper will present the numerical approaches employed to predict sensor reaching radiances and comparisons with analytically predicted results. Representative data sets generated by the DIRSIG model for a topographical LIDAR will be shown. Additionally, the results from phenomenological case studies including standard terrain topography, forest canopy penetration, and camouflaged hard targets will be presented.
\end{abstract}

Keywords: DIRSIG, laser radar modeling, ladar, lidar, topographic lidar

\section{INTRODUCTION}

\subsection{Model History}

The initial development of the Digital Imaging and Remote Sensing Image Generation (DIRSIG) model was begun at Rochester Institute of Technology (RIT) in the late 1980's as a 3D simulation environment for predicting images that would be produced by thermal infrared systems. Since that time, the model has been expanded to cover the 0.35 to 20.0 micron region of the spectrum. The model is designed to produce passive broad-band, multi-spectral and hyper-spectral imagery through the integration of a suite of first principles based radiation propagation modules. These object oriented modules address tasks ranging from bi-directional reflectance distribution function (BRDF) predictions of a surface, to time and material dependant surface temperature predictions, to the dynamic viewing geometry of scanning imaging instruments on agile platforms. ${ }^{1}$ In addition to the myriad of DIRSIG specific objects that have been created, there is a suite of interface objects that leverage externally developed components (e.g. MODTRAN ${ }^{2}$, FASCODE, THERM $\left.^{3}\right)$ that are modeling workhorses for the multi- and hyper-spectral community. The software is employed internally at RIT and externally within the user community as a tool to aid in the evaluation of sensor designs and to produce imagery for algorithm testing purposes. Key components of the model and some aspects of the models overall performance have been gauged by several validation efforts over the past decade of the model's evolution. ${ }^{4,5}$

\subsection{Historical Modeling Approach}

The modeling philosophy that has driven the DIRSIG model development over the years is one that favors firstprinciples radiation transfer mechanisms over statistical or empirical modeling approaches. Most statistical or empirical models have been derived from specific data sets that feature specific conditions. The fit of these models to the

\footnotetext{
${ }^{\dagger}$ Send correspondence to Scott Brown at brown@cis.rit.edu

* Send correspondence to Daniel Blevins atddb1190@rit.edu
} 
respective original data may be exceptional, but depending on the underlying methodology employed, the model may not be applicable at for a different time of day, for a different season, for different sensor, etc. Such models may have a high degree of accuracy for specific cases, but very little flexibility for modeling others. These same limitations may be applicable to some classes of statistically based models. In contrast, the approach that is employed by the DIRSIG tool is to model as many physically based interactions as possible by utilizing model inputs that predominately consist of geometric, optical and thermodynamic inputs. The underlying radiative transfer model then interacts with this combined geometric and optical scene model to predict the radiational flux into a given direction for a specific set of conditions. Although this approach may not model a specific data set as accurately as an empirical or statistical that is derived from the imagery, this modeling approach has a higher degree of flexibility by allowing the user to change the imaging conditions, scene conditions, etc.

The general approach of mating high fidelity geometric models with high fidelity optical and thermodynamic models prevails throughout the DIRSIG model. Some empirically and statistically drive models appear in the lower levels of the overall model, but the higher level modeling capabilities are derived from the integration of a myriad of lower level representations. The authors have found this modeling architecture capable of reproducing specifically sought phenomenology as well as unexpected collateral phenomenology that might not be realized using other techniques. The images in Figure 1 visually illustrate some of the spatial and spectral fidelity resulting from the modeling approach used by the DIRSIG model for an urban scene of Rochester, NY.

\subsection{Active System Justification}

Up until 2002, the DIRSIG model was focused on simulating passive multi-spectral and hyper-spectral sensing systems in the visible through thermal infrared regions. At that time, the ability to perform trade studies between passive and active laser radar systems would require the use of separate modeling packages that may use different geometric, optical and thermodynamic descriptions. In addition, the available state-of-the-art LIDAR scene simulation tools did not support rigorous atmospheric interactions, participating mediums, multiple bounce/scattering, thermal and reflective region passive returns, complex scene geometries, moving platform and scanning effects, detailed material optical descriptions (BRDF and scattering models) and time-gated returns. The benefits of an integrated active LIDAR and passive multi- and hyper-spectral passive simulation environment that leverage a unified set of model inputs and underlying radiation propagation models would be significant. A prototype of this combined simulation environment was completed by Burton and Brown in 2002. ${ }^{6,7}$ This paper discusses the fully developed model available in the current version of the DIRSIG scene simulation software with respect to topographic mapping applications. This simulation tool allows users to simulate active and passive sensors using the same scenes and scenarios and allow for the evaluation of passive versus active approaches to specific problems and the exploration of data fusion between these two imaging paradigms.
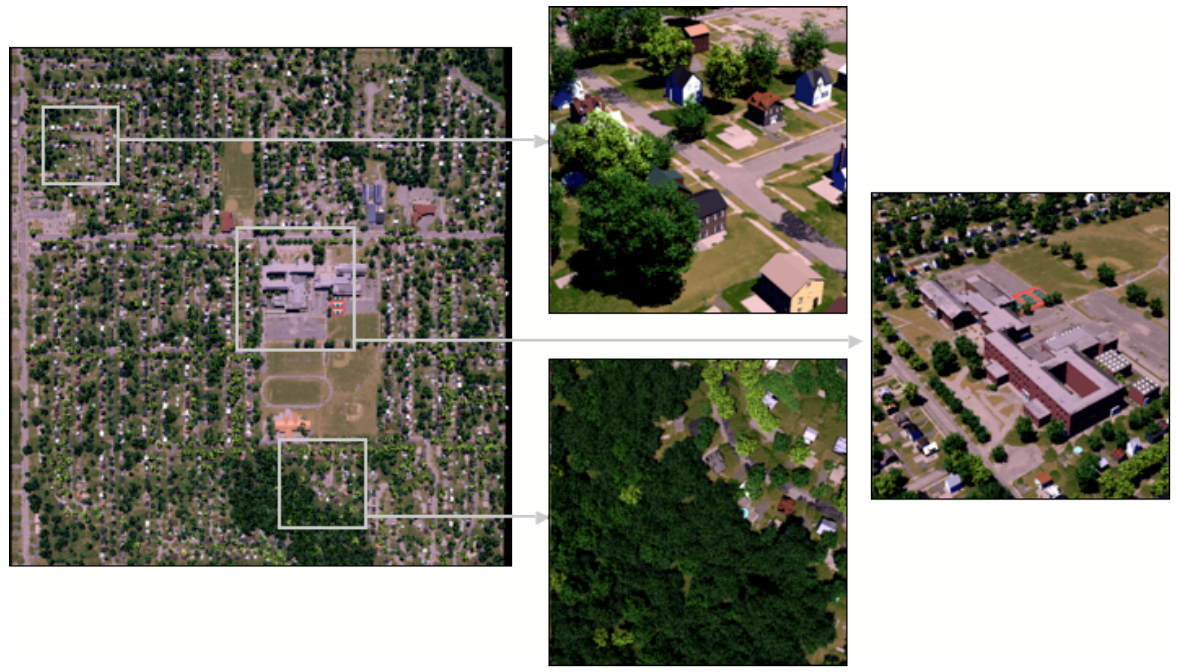

Figure 1: RGB images generated by the DIRSIG model of an urban residential scene illustrating the spatial and spectral fidelity of these scene models. 


\section{MODEL DESCRIPTION}

\subsection{Numerical Modeling}

The addition of an active, laser radar capability to the DIRSIG model was accomplished by the addition of a suite of new objects to the existing radiometry framework. In general, the model is designed to predict the returned fluxes from the scene as a function of time with respect to the shooting of the source laser. The specific challenges of this imaging model were driven by the requirement to predict the received photon counts as a function of space and time. The photon flux arriving at a LIDAR system often approaches discrete photon events due to the low amount of backscattered radiation and can prove difficult for many traditional Monte-Carlo ray tracing techniques. Additionally, the temporal structure of these returns is driven by the spatial structure of the scene and the total travel time of arriving photons accrued during multiple bounce and scattering events within the scene. Analytical, statistical, and existing passive radiometry solvers were found to be insufficient in many instances, particularly for low flux situations. Thus, a new approach was sought.

\subsubsection{Modified Photon Mapping}

The new approach that was identified leverages a modeling technique called photon mapping. ${ }^{8}$ Although the original presentation focused on photon mapping as a monochromatic approach, various strategies have been introduced to handle spectral sources using this methodology. One reason that this technique was selected was because photon mapping has been demonstrated to be applicable to traditional solid geometry reflective illumination and scattering and absorption by participating mediums, particularly in multiple bounce and multiple scattering cases.

The photon mapping approach is a hybrid of traditional forward and backward Monte-Carlo ray tracing techniques. In this two-pass method, source photons are shot from a source into the scene using forward ray tracing during the first pass and then collected using a backward ray tracing during the second pass (Figure 2). The collection or rendering process utilizes the events recorded during the first pass to calculate the sensor reaching radiance. For the purpose of active laser radar applications, some modifications to the basic photon mapping treatment were made including the tracking of total travel time and a literal photon counting process.

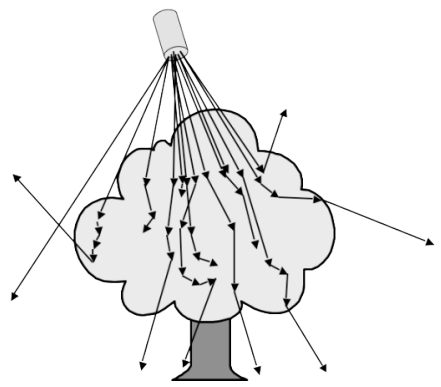

(a) Pass 1 - Photon Tracing

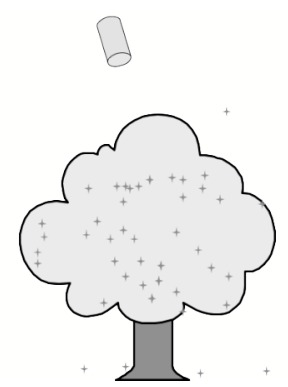

(b) Recorded Photon Map

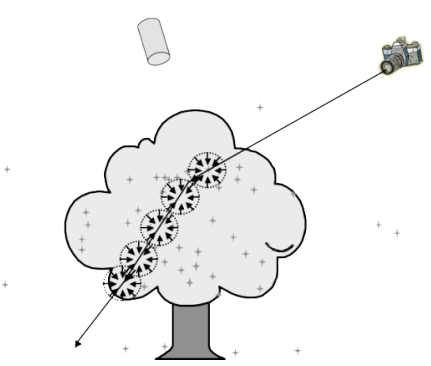

(c) Pass 2 - Photon Collection

Figure 2: Illustration of core photon mapping concepts which are (a) forward propagation of photons, (b) the resulting photon map, and (c) using photon map to predict received photon counts at the detector.

During the first pass, a modeled photon is cast into the scene from the source and performs a pseudo-random walk through the scene based on the optical properties of the surfaces or mediums that it encounters. At the location of each interaction, information regarding the interaction event is stored into a fast, 3D data structure or map (e.g. a kdtree $\mathrm{e}^{9,10,11}$ ) that can be queried during the second pass. A critical addition to traditional photon mapping paradigm was a field to track each photon's total travel time. This time field accrues the travel time for all of the multiple bounce/scattering events to be recorded and is used for time gating during the second pass. The modeled photon is followed until it is absorbed by some element within the scene. The photon casting process is repeated until a specified number of interaction events have been recorded in the map. The characteristics of the laser source are incorporated into the spatial, spectral and temporal distribution of the modeled photons fired into the scene. 
From an absolute radiometry perspective, each modeled photon represents a "photon bundle" emitted from the source. Much like traditional photon mapping, the variance of the estimated received photon stream is intimately linked to the number of photon bundles cast into the scene. The number cast must be sufficient to obtain a statistically significant number of events throughout the field-of-view of the sensor. Depending on the angular extent of the beam, the field-of-view of the sensor and the spatial detail of the scene approximately 250,000 to $1,000,000$ photons can be utilized in the photon mapping process to create reliable statistics for topographic returns. For volume scattering returns, this number may need to be higher depending on the absorption and scattering coefficients and the spatial extent of the participating volumes.

For the second pass, rays are shot from the image plane into the scene until a scene element is intersected. Traditional photon mapping approaches utilize the localized photon map density to estimate the incident irradiance at a surface or volume element. For this application, the photon map is used literally as a photon counting mechanism. The model queries the 3D data structure that was filled during the first pass to find a set of incident photons arriving at the surface (or volume element in the case of a participating medium). Each of these photon bundles is individually redirected towards the sensor using the applicable surface reflectance or volume scattering coefficients for the specific incident and excitant geometry (Figure 3). The volume over which the photons are collected is based upon the detector's field-of-view and a user-defined spatial oversampling factor. The received photon bundles are then temporally distributed (based on the bundle's parametric temporal shape) and quantized based upon the user-defined listening window and sampling frequency. The result is a time-gated data cube for the listening window that contains the received number of photons at each sampling interval for each detector element.

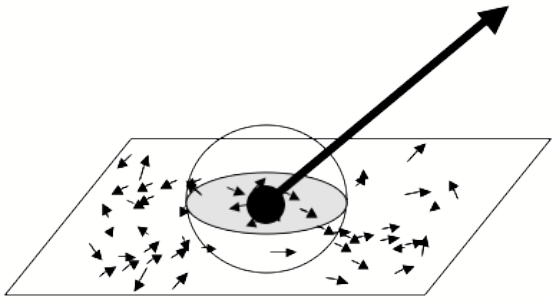

(a) Surface

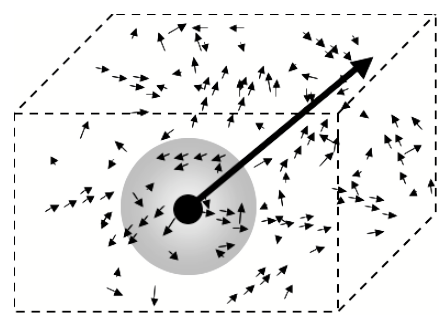

(b) Volume

Figure 3: Modified photon mapping photon count estimate and projections.

\subsection{Model Implementation}

The integration of photon mapping into the DIRSIG model entailed the implementation of several new objects. The first was the basic support for photon mapping. This entailed the implementation of a 3D data structure that can be quickly searched using spatial queries and the creation of a photon object that would be propagated and stored into the photon map. For this purpose, the traditional kd-tree was used. The next object was a flexible source model that could support directional characteristics and the spatial, spectral and temporal distribution of the source photons. In the current implementation, the system is modeled in a monochromatic mode at the peak wavelength of the source. The temporal shape of the pulse is stored parametrically in each photon rather than shooting photons as a function of time. The pointing and spatial distribution of the source is numerically modeled based on either Gaussian or top-hat spatial distributions. The LIDAR support allows the user to model co-axial and bi-static systems by providing nearly arbitrary relative source to detector positioning and pointing geometries.

\subsubsection{Forward Propagation}

The photon shooting function leverages the generic ray tracing support that already existed within the DIRSIG model. The ray tracer interacts with scene elements that have material specific properties. Each material has a set of surface optical properties and an optional set of bulk or medium properties. The surface properties include a spectral reflectance and/or emissivity property. The currently supported reflectance (BRDF) models include importance based sampling functions to support the forward and backward Monte-Carlo ray tracing. The bulk properties include spectral extinction, absorption and scattering coefficient models. When volume scattering is modeled, a phase function object can also be configured to describe the directional nature of the scattering. The phase function objects also support importance based sampling functions to support the forward and backward Monte-Carlo ray tracing. Although these optical 
descriptions existed prior to the addition of the LIDAR capabilities, their interfaces were enhanced during this effort to facilitate an efficient implementation of the photon mapping subsystem.

\subsubsection{Backward Propagation}

The next major component of the model to be implemented was the collection of photons from the photon map and forward propagation into the imaging system. The modeling of the radiometric returns from a scene element is handled by a class of objects in DIRSIG referred to as radiometry solvers. A radiometry solver encapsulates an approach to predicting the energy reflected, scattered and emitted by a surface or volume. For example, there already existed a specific solver for predicting the passive returns from "hard" surfaces and another for a volume of gas (e.g. plume). These solvers utilize the material specific surface and bulk optical properties to predict their results. One or more radiometry solvers can be assigned to each element in the scene. To support the active LIDAR returns, a new radiometry solver was created to compute the returns from a scene surface or volume by using the optical properties and the photon map to estimate the number of incident photons at the element's point in space. Unlike the existing passive radiometry solvers that would place the final result in a time-independent result object, the LIDAR-specific radiometry solver places the result into a time-gated result object. The time gating is based on a user-defined signal gate consisting of a start, stop and delta time.

The final component was the implementation of a "capture method" object within the system that directs how outputs for the detectors on the focal plane are computed. The LIDAR specific capture method developed under this effort backward propagates rays from each detector element into the scene where it intersects scene elements, runs the appropriate radiometry solvers (passive and active), forward propagates the energy to the focal plane and then writes the arriving photons counts to the output file.

\subsubsection{Atmospheric Returns}

At this time the atmosphere is assumed to be spatial uniform as a function of position and altitude, which may not be accurate for some real-world applications. The horizontal and vertical structure of the atmosphere results in different absorption and scattering characteristics as a function of location. Ideally, robust atmospheric optical models like MODTRAN and FASCODE would drive both the extinction and scattering optical properties of the atmosphere. At this time, the extinction coefficients used by the DIRSIG model are extracted from the existing MODTRAN and FASCODE derived tables. However, extraction of the vertically structured scattering coefficients and phase functions from MODTRAN (FASCODE does not support scattering) would require custom modifications to the MODTRAN code. For the time being, the user can create their own scene elements with user-defined extinction and scattering properties to replace the atmospheric if this level of control is critical.

Under most conditions, the extinction and backscatter coefficients of the atmosphere are extremely small, which means that the probability of absorption and scattering events within the atmosphere is very low. For example, the scattering coefficient for a dry atmosphere might be $1 \times 10^{-5}[1 / \mathrm{m}]$, which means that you would need to shoot $10^{5}$ photons into a 1-meter long box of atmosphere to witness a one scattering event. Many systems are attempting to resolve vertical resolutions of a fraction of a meter and from an altitude of several thousand meters, which implies that you would need to model $10^{10}$ photons within each spatial detector element in order to get a one scattering event within each numerical contribution element. To achieve robust statistics, this number would be ideally several orders larger.

To use the numerical approach utilized by the photon mapping technique, the number of photons that would need to be shot into the atmosphere to create a statistically accurate representation of the scattering events would be many of orders of magnitude larger than the number of photons needed to model the topographic returns. To avoid the problems of predicting the atmospheric returns numerically, the atmospheric returns from the model are currently modeled analytically using the formulation proposed by Measures. ${ }^{12}$

$$
P\left(\lambda_{L}, R\right)=P_{L} \frac{c \tau_{L}}{2} \frac{A_{r}}{R^{2}} \xi\left(\lambda_{L}\right) \xi(R) \beta\left(\lambda_{L}, R\right) \exp \left[-2 \int_{0}^{R} \sigma_{e x t}\left(\lambda_{L}, r\right) d r\right]
$$

where $P\left(\lambda_{L}, R\right)$ is the total scattered laser power received in watts, $\lambda_{L}$ is the peak wavelength of the laser in meters; $\mathrm{R}$ is the range of the contribution element in meters; $P_{L}$ is the average power of the laser pulse in watts; $c$ is the speed of light in meters per second, $\tau_{L}$ is the pulse length in seconds; $A_{r} / R^{2}$ is the acceptance solid angle of the receiver optics with a collecting area $A_{r} ; \xi\left(\lambda_{L}\right)$ is the receiver optics transmission at the laser wavelength; $\xi(R)$ is the geometrical 
form factor at range $\mathrm{R} ; \beta\left(\lambda_{L}, R\right)$ is the atmospheric backscatter coefficient at the laser wavelength and range $\mathrm{R}$ in inverse meters and $\sigma_{e x t}\left(\lambda_{L}, R\right)$ is the atmospheric total extinction coefficient in inverse meters. For most of the topographic systems modeled to date, the atmospherically scattered photon returns amount to only a few photons accumulated over the entire path length, which is far below the detection level of the modeled systems. However, if the tools is used to model a significantly longer path length or an optically thicker atmosphere (containing fog), then these numbers will grow to be large enough for consideration by the detection model.

\subsubsection{Instrument and Platform Modeling}

Most operational laser radar instruments are flown on aircraft and utilize some method of aircraft relative scanning to increase the spatial coverage of the system. The changes in viewing geometry during the scanning process and the location, orientation and stability of the instrument platform can affect the final data products. For example, the ability to resolve a specific object in a topographic data product derived from a dataset might be dependant on the angle from which it is illuminated. The overall accuracy of a derived topographic product might depend on the overall stability of the platform and knowledge of the platform's position. The DIRSIG model has a flexible platform model that allows the platform to be positioned and oriented as a function of time. Furthermore, the instrument can be pointed with respect to the platform either statically or dynamically using one of the available instrument mount objects. These mount objects can support temporal scanning including basic sinusoidal across-track scanning as a function of a user-defined scan rate.

\subsubsection{Generalized Modeling Process}

With the basic components of the model now described, the overall modeling process can now be summarized. A modeling run consists of the user specifying the scene to be modeled, the instrument and instrument mount description, the source description, the platform positioning data, and a set of tasks that describe time windows over which data is to be generated. The data generation process begins by walking through each user-define task according to a step time that is usually driven by the source pulse rate. During each time step, the platform and instrument mount positions and orientations are computed for the current time, the source is fired which fills the photon map, the focal plane is captured which collects the photons and propagates them to the sensor, and the focal plane reaching photon counts are written to the output file. This cycle repeats for each time in the task window and for each task in the list.

The final product of the DIRSIG tool is a 3D cube consisting of photon counts as a function of two horizontal spatial dimensions and one temporal dimension (see Figure 4). Typically, the resulting data cube is ingested by an external focal plane model to handle instrument specific detection schemes (e.g. linear mode versus Gieger mode), noise sources, etc. In these situations, the data cube should be generated with a significant amount over oversampling in the spatial and temporal dimensions to allow for spatial and temporal integration by the external sensor model. Further external processing of the resulting modeled raw instrument outputs can be used to create final data products (e.g. topographic maps, gas detection maps, etc.).

\section{MODEL DEMONSTRATION AND ANALYTICAL RESULTS}

Although the photon mapping concept theory is rooted in the underlying physics of the radiative transfer process as outlined in Section 2 and has produced visually appealing results for images in the computer graphics community, the question remains as to whether the approach can produce radiometrically accurate results, particularly for a participating medium. The next few subsections leverage a multi-pronged verification approach with simplified analytical scenarios, model-to-model comparisons, and sample phenomenological case studies. One should note that verification indicates a proper physical basis such that the results are consistent with the underlying physics. 


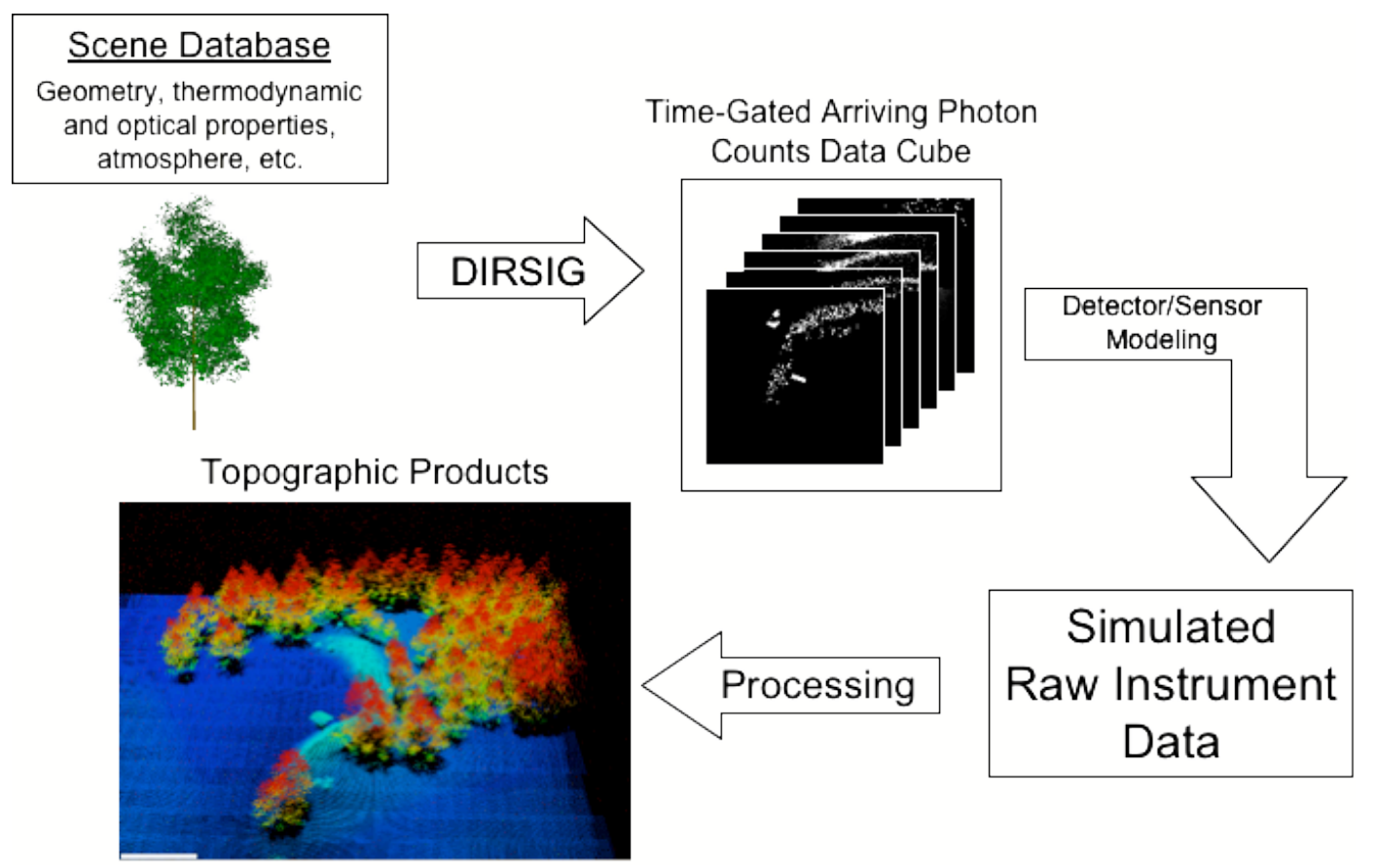

Figure 4: An overview of an end-to-end system simulation using DIRSIG for data generation.

\subsection{Analytical Comparisons}

\subsubsection{Simple Reflective Surface}

The governing equation for the received signal captured by the sensor is derived from the general LIDAR equation for elastic scattering. Based upon Measures ${ }^{12}$, the received number of photons from a range $R$ due to elastic backscattered radiation can be written as:

$$
N_{\text {detected }}(\lambda, R)=N_{L}(\lambda) \xi(\lambda, R) \frac{A_{r}}{R^{2}} \frac{\rho_{s}(\lambda)}{\pi} \times \exp \left[-2 \int_{0}^{R} \sigma_{e x t}(\lambda, r) d r\right]
$$

where $\xi(\lambda, R)$ is a system function determined by the geometric considerations of the receiver optics, the quantum efficiency of the detection system at each wavelength, and the overlap between the transmitted laser beam and the field of view of the receiver; $A_{r} / R^{2}$ is the acceptance solid angle of the receiver optics with a collecting area $A_{r} ; N_{L}(\lambda)$ is the average number of photons in the transmitted pulse at wavelength $\lambda ; \sigma_{\text {ext }}(\lambda, r)$ is the extinction coefficient of the participating medium at the wavelength $\lambda$ for the range $r ; \rho_{s}(\lambda)$ is the backscattering efficiency at of the target surface. $\xi(\lambda, R)$ is usually assumed to be separable into a wavelength dependent weighting; $\xi(\lambda)$, and a geometrical form factor, $\xi(R) . \xi(\lambda)$ is dependent primarily upon the receiver design characteristics and for the purposes of this subsection shall be assumed to be unity whereas typical values range from 0.5 to 0.8 . The geometrical form factor is used to adjust the equation to account for a variety of factors and is often fairly difficult to evaluate for a real LIDAR system. If one assumes that the laser power distribution is Gaussian in the target plane and that the limiting aperture is the detector size instead of the telescope objective lens (or mirror), then the LIDAR system function can be expressed as

$$
\xi(\lambda, R)=\xi(\lambda) \frac{1}{\pi W^{2}(R)} \frac{R^{2}}{\pi r_{0}^{2} f^{2}} \int_{A_{D}} \int_{A\left(r, \psi, \gamma_{0}\right)} \exp \left[-\frac{r^{2}}{W^{2}(R)}\right] d A d A_{D}
$$

where $f$ is the effective focal length of the system, $r_{0}$ is the radius of the receive telescope aperture, $R$ is the range, $W(R)$ is the transmit beam waist radius at range $R, A_{D}$ is the area of the detector in the focal plane, and $A\left(r, \psi, r_{0}\right)$ is a circle of 
radius $r_{0}$ that is centered at $(r, \psi)$ in the target plane. When Equations 2 and 3 are combined, the topographic LIDAR equation can be rewritten as

$$
N_{\text {detected }}(\lambda, R)=N_{L}(\lambda) \xi(\lambda) \frac{\rho_{s}(\lambda)}{\pi} \times \exp \left[-2 \int_{0}^{R} \sigma_{e x t}(\lambda, r) d r\right] \times \frac{1}{\pi W^{2}(R)} \frac{1}{f^{2}} \int_{A_{D}} \int_{A\left(r, \psi, r_{0}\right)} \exp \left[-\frac{r^{2}}{W^{2}(R)}\right] d A d A_{D} .
$$

Using Equation 4 and the system parameters described in Table 1, one can analytically evaluate the number of photons detected from a surface. To minimize atmospheric effects, a dry atmosphere will be assumed with the extinction coefficient of $1 \times 10^{-5}[1 / \mathrm{m}]$. The planar surface is Lambertian with a known reflectance and is $1200 \mathrm{~m}$ downrange from the co-located transmitter and receiver.

Table 1. LIDAR system configurations for DIRSIG verification runs

\begin{tabular}{|l|c|}
\hline \multicolumn{2}{|c|}{ Parameter } \\
\hline \hline Wavelength $(\lambda)$ & $532 \mathrm{~nm}$ \\
\hline Pulse Energy $\left(P_{L}=N_{L} h c / \lambda\right)$ & $6 \mu \mathrm{J}$ \\
\hline Pulse Repetition Frequency (PRF) & $5 \mathrm{KHz}$ \\
\hline Pulse Length $\left(\tau_{D}\right)$ & $1 \mathrm{~ns}$ \\
\hline Aperture Radius $\left(r_{0}\right)$ & $100 \mathrm{~mm}$ \\
\hline Focal Length $(f)$ & $400 \mathrm{~mm}$ \\
\hline Transmit Beam Radius $\left(w_{0}\right)$ & $5 \mathrm{~mm}$ \\
\hline Beam Divergence $\left(\phi_{D}\right)$ & $8.0 \mathrm{mrad}$ \\
\hline Detector Size & [Varied from $100 \mu \mathrm{m}$ to $50 \mathrm{~mm}^{* *}$ ] \\
\hline$* * 50$ mm detector represents collection of entire beam
\end{tabular}

Figure 5(a) displays both the analytical prediction dictated by Equation 4 and DIRSIG's integrated output for a single pixel versus the surface reflectance. As shown in Figure 5(a), the analytical and numerical results are well correlated and exhibit appropriate behaviors. Figure 5(b) is a plot of the impact of the detector size on the number of photons detected from the Lambertian surface. Once the detectors are collecting the majority of the transmit beam (detector pitch $>3 \mathrm{~mm}$ ), then the number of photons detected varies linearly with the surface reflectance. However, when the detector sizes are such that the receive fields-of-view are small compared to the transmit beam footprint, the geometrical form factor has a greater impact on overall number of detect photons and an incremental increase in detector size results in a dramatic improvement.

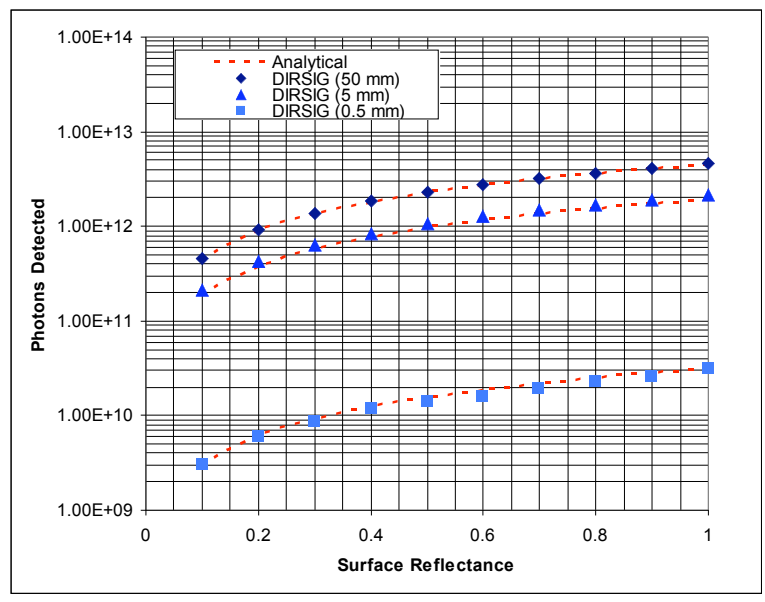

(a)

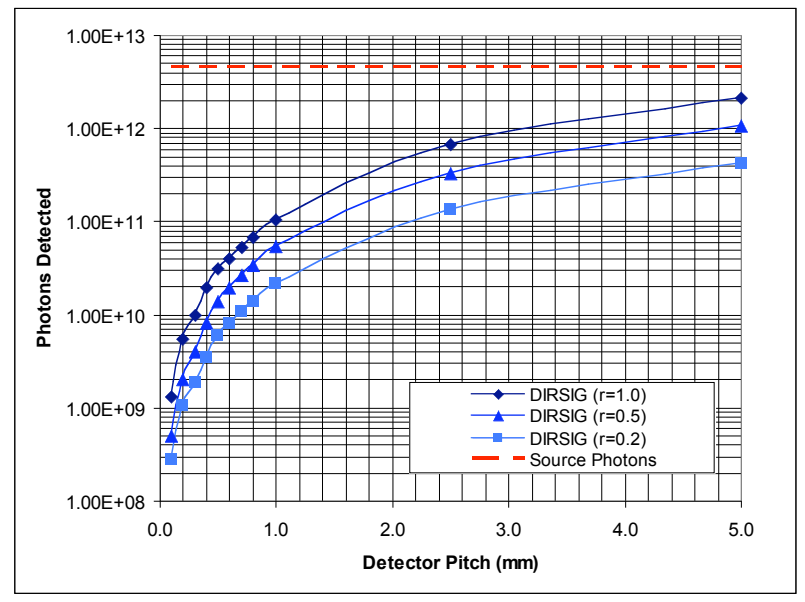

(b)

Figure 5: (a) DIRSIG integrated number of photons detected from a Lambertian surface of varying reflectance at $1200 \mathrm{~m}$ downrange for a single pulse. (b) Detector size impact on the integrated number of photons detected from a Lambertian surface of varying reflectances at $1200 \mathrm{~m}$ downrange for a single pulse. (Note that $50 \mathrm{~mm}$ detector size used to collect entire beam, presuming a detector limiting aperture). 


\subsection{Phenomenology Demonstrations}

\subsubsection{Tree Crown Simulation}

The first phenomenology demonstration will address laser radar returns from a tree crown. For this example, a single tree is modeled and the temporal structure of the reflected photon counts will be explored. The geometry of the tree was created using a software package called OnyxTREE and the surfaces were attributed with leaf spectral reflectance and transmission measurements made at RIT. The OnyxTREE software is capable of producing physically realistic tree geometries using detailed descriptions of tree growth patterns. ${ }^{13}$

Figure 6 includes a 3D photon map density plot resulting from a single pulse modeled with 1,000,000 photon bundles. In the plot of the photon map, the shape and detail of the tree crown can be seen as well as the resulting shadow on the ground. Within the tree shadow there are some areas with increased photon counts that are evidence of direct illumination via foliage "poke through" and indirect illumination due to multiple reflections. The plot of the photon counts as a function of time/distance in Figure 6 results from spatially integrating a region encompassing most of the tree (refer to the highlighted box in the photon count frames). This magnitude vs. time plot shows a steady decay within the tree crown due to absorption and reflection, the late arrival of photons from ranges between the crown and the ground due to multiple bounces within the crown and the ground return itself. The magnitude of the ground return indicates a non-trivial probability of photons reflecting off the ground beneath the tree itself. The magnitude of this return would be a function of the tree's optical properties and leaf density (leaf area index). The images in the lower portion of Figure 6 are temporal slices of the photons counts arriving at the sensor. These spatial count density maps reveal horizontal and vertical structure of the tree itself that can be utilized to reconstruct the tree height and shape. The modeling of a tree canopy (instead of a single tree) is included in the end-to-end scene simulation at the end of this paper.

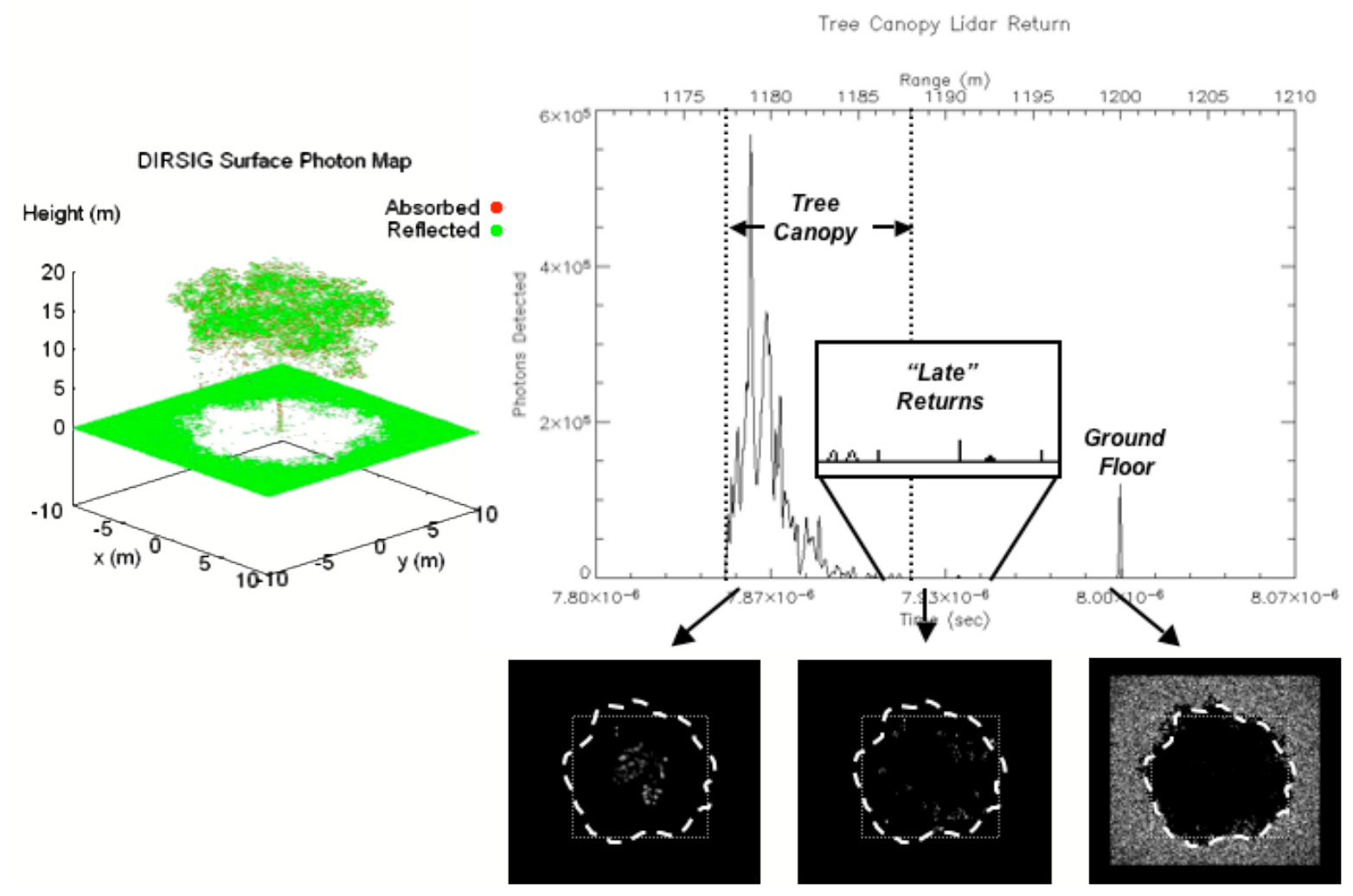

Figure 6: A demonstration of a single, deciduous tree. The 3D photon map (top left) shows the density of photon reflection and absorption effects. The plot captures the spatially integrated photon counts as a function of time/range for an area encompassing the tree (refer to the highlighted box in the lower frames). The photon count frames (bottom right) represent the spatial density of the photons arriving at the sensor for specific times/ranges. 


\subsubsection{Camouflaged Vehicle Simulation}

Another common application area for topographic laser radar systems is for camouflage penetration problems. To demonstrate the use of the DIRSIG model for this task, a small scene was constructed that contains a HMMWV ("humvee") under a camouflage net held in place by supports on a terrain (Figure 7, top left). The camouflage net is modeled as a continuous surface that has holes cut into it using a high spatial resolution "hole mask" that introduces geometric transmission due to spatial variations in fill factor. The solid areas of the net are attributed with a set of three fabric materials that have different reflectance and transmission factors. The vehicle and surrounding terrain is also fully attributed with appropriate surface optical properties.

The DIRSIG model produced a highly oversampled, time-gated, photon count cube for this scene. For visual reference, the DIRSIG model also produced the height "truth map" shown in the top right of Figure 7 that illustrates the amount of camouflage "poke through" that can be expected and the visibility of net supports and vehicle underneath. The series of images at the bottom of Figure 7 show the spatial photon densities as a function of time. At this high temporal sampling rate, small differences in vertical structures can be easily resolved including variations in the net surface, the net support, the roof and hood of the vehicle and the terrain beneath. The ability to model scenes with these complex interactions and surface properties accentuates the benefit of physics based approach over what analytical or statistical models can provide.

\subsubsection{End-To-End Topographic Mapping Demonstration}

The end-to-end modeling of operational systems entails using the DIRSIG model to create time-gated photon counts that can be processed by an external sensor model to produce simulated raw instrument data. These simulated instrument data sets can then be used with conventional data processing tools to create topographic data products. For this demonstration, a data collection of the ALIRT system developed at MIT Lincoln Laboratories was simulated over a small scene located on the RIT campus. The DIRSIG scene database used in this end-to-end simulation was originally developed for passive, tower based collections for the purpose of camouflage and landmine algorithm testing. The entire scene is approximately $300 \times 300$ meters and contains terrain, trees, and man-made elements. The operational ALIRT system was flown during the winter of 2004 over the corresponding portion of the RIT campus to capture reference data sets for future validation efforts. The original scene database was modified to reflect the scene as it appeared during the data collection by the actual ALIRT sensor.

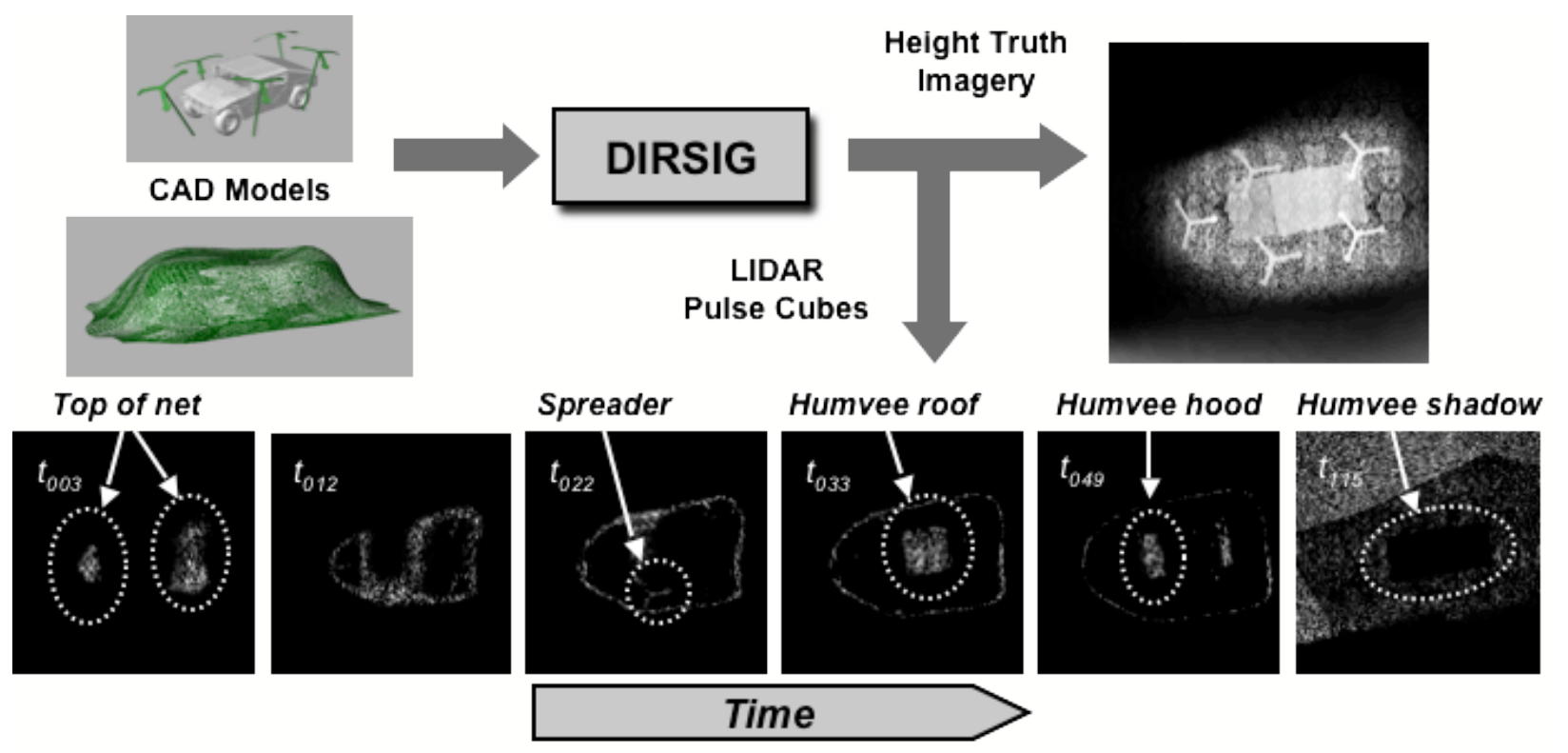

Figure 7: A demonstration of a HMMWV under a camouflage net (top left). The height truth map (top right) illustrates features that are reproduced in the photon count time/range sequence (bottom). 

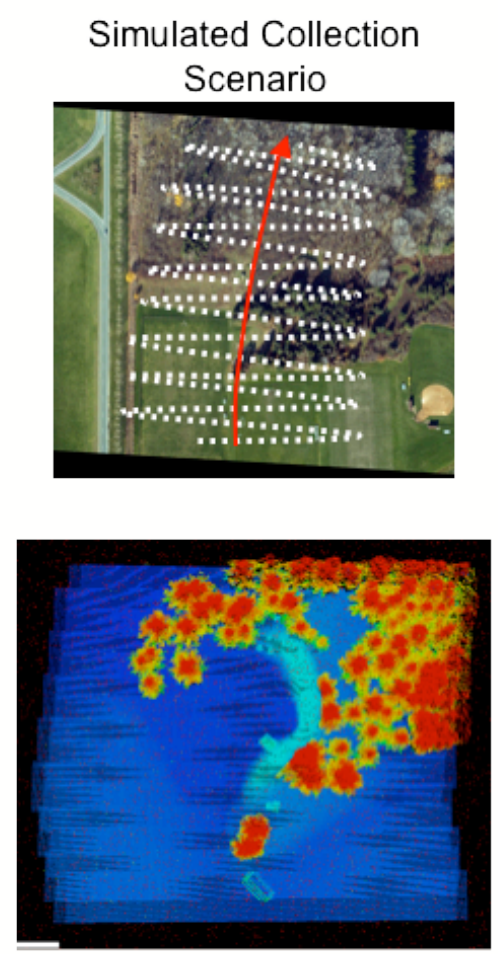

Derived Topographic Product (Overhead)

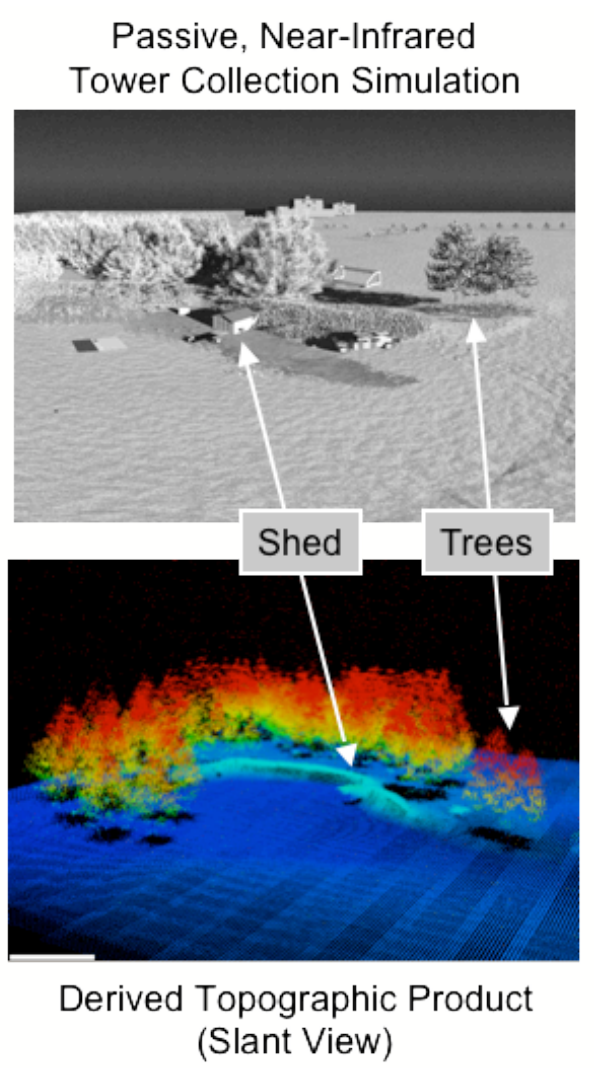

Figure 8: The end-to-end simulation of the ALIRT sensor included flight data from an actual over-flight of the scene and the acrosstrack scanning of the system (top left). The general scene in the near-infrared from a tower-based instrument (top right). The derived topographic products viewed from overhead (bottom left) and a slant view (bottom right). Note, that scene elements were removed for the active simulation to reflect the scene acquired by the actual ALIRT instrument for future validation efforts. Topographic reconstructions courtesy of ITT Industries, Space Systems Division.

The ALIRT instrument uses a spatial array that is scanned in the across-track direction from an airborne platform. The across-track scan speed is slower than the laser pulse repetition rate so that the collected area over laps significantly from pulse to pulse. The ALIRT instrument and collection characteristics were modeled using laser and instrument properties supplied by MIT Lincoln Laboratories. The simulated data cubes produced by the DIRSIG model utilized the flight data from the actual ALIRT over-flight of the scene. The resulting time-gated, photon count cubes were then processed using a sensor model developed by ITT Industries, Space Systems Division, that features a rigorous treatment of the ALIRT focal plane and instrument. The resulting simulated raw instrument data streams were then post-processed using QT Viewer (developed at Johns Hopkins Applied Physics Laboratory) to view the resulting topographic products (see Figure 8). The scan sweeps of the system are clearly visible in the overhead and slant topographic projections. These artifacts arise due to the noise-free knowledge of the platform location and platform relative pointing of the instrument during scanning. Future simulations will include a noise term on these quantities to introduce the inherent uncertainty in these values.

\section{SUMMARY AND CONCLUSIONS}

The extension of the DIRSIG model from passive-only scene simulations to the generation of active laser radar simulations was presented. The numerical modeling approach utilized by the tool allows for the simulation of complex scenes and in-scene interactions that cannot be predicted using common analytical or statistical approaches. This capability was achieved by extending the traditional photon mapping approach to track the travel time of photons. The model results were compared to analytical predictions for specific source and topographic scene scenarios to verify the 
absolute radiometry of the model implementation. The model was also exercised to reproduce common phenomenology including multiple bounce and foliage penetration. Finally, the model was demonstrated in the context of an end-to-end topographic system model by simulating the collection of a real sensor (including platform motion and instrument scanning) over a scene with significant spatial complexity.

A rigorous validation of this DIRSIG LIDAR model for topographic applications is currently underway using real world data collected by the ALIRT system. Other future efforts will focus on the treatment of volume scattering and absorption within this modeling approach. The support for these radiative transfer mechanisms is already included in the model and is currently under evaluation for use in simulating Differential Absorption LIDAR (DIAL) systems. Future modifications may include tracking the polarization state, the phase distributions and the spectral structure of the photons represented by each modeled photon stored in the photon map. At this time, all interactions within the model are elastic in nature, but the incorporation of inelastic collisions can be handled within this treatment by imposing a combination of wavelength and temporal shifts during each interaction.

\section{ACKNOWLEDGMENTS}

The authors wish to thank Norman Lopez, William Cook, Raymond Lines and James Michel of ITT Industries, Space Systems Division, Rochester, NY and MIT Lincoln Laboratories for their support and contributions to this effort.

\section{DISCLAIMER}

The views expressed in this article are those of the author and do not reflect the official policy or position of the United States Air Force, Department of Defense, or the U.S. Government.

\section{REFERENCES}

1. Schott, J.R., Brown, S.D., Raqueño, R.V., Gross, H.N., \& Robinson, G. "An advanced synthetic image generation model and its application to multi/hyperspectral algorithm development", Canadian Journal of Remote Sensing, Vol. 25, No. 2, June 1999.

2. Berk, A., L. S. Berstein, and D.C. Robertson, "MODTRAN: a moderate resolution model for LOWTRAN 7", GLTR-89-0122, Spectral Sciences, Burlington, MA, 1989.

3. DCS Corporation, "AIRSIM thermal signature and prediction analysis tool definition and analysis of object inputs", DCS Technical Note 9090-002-004, DCS Corporation, 1990.

4. Mason, J.E., Schott, J.R., and Rankin-Parobek, D., "Validation analysis of the thermal and radiometric integrity of RIT's synthetic image generation model, DIRSIG", Proc. of the SPIE, Characterization and Propagation of Sources and Backgrounds, Vol. 2223, pp. 474-487. Orlando, FL, 1994.

5. Brown, S.D., Schott, J.R., Raqueño, R.V., Kraska, T., White, R., "Validation and Analysis of the Digital Imaging and Remote Sensing Laboratory's Synthetic Image Generation Model, DIRSIG", Proc. of the Ground Target Modeling and Validation Conference, Houghton, MI, August 1996

6. Burton, R., John R. Schott and Scott D. Brown, "Elastic LADAR Modeling for Synthetic Imaging Applications", Proc. of SPIE, Vol. 23, No. 13, pp 144-155, 2002

1. Burton, R., "Elastic LADAR Modeling for Synthetic Imaging Applications", Ph.D. thesis, Rochester Institute of Technology, Rochester, NY, 2002.

7. Jenson, H.W. Realistic Image Synthesis Using Photon Mapping, A.K. Peters, Ltd., Natick MA, 2001

8. Bentley, J.L, "Multidimensional binary search trees used for associative searching", Communications of the ACM, Vol. 18, No. 9, pp 509-517, 1975

9. Bentley, J.L, "Multidimensional binary search trees in database applications", IEEE Trans. On Soft. Eng., Vol. 5, No. 4, pp 333-340, July 1979

10. Bentley, J.L, and J.H. Friedman, "Data structures for range searching”, Computing Surveys, Vol. 11, No. 4, pp 397409, 1979

11. Measures, R. M., Laser Remote Sensing: Fundamentals and Applications, John Wiley \& Sons, Inc., New York, 1984

12. OnyxTREE Software, information available online at http://www.onyxtree.com 